\title{
Simulation of Granular Compacts in two dimensions
}

\author{
A.M. Vidales ${ }^{1}$, V. M. Kenkre ${ }^{2}$ and A. Hurd \\ 'Departamento de Fisica y CONICET, Universidad Nacional de San Luis, 5700 San Luis, Argentina. \\ ${ }^{2}$ Center for Advanced Studies, Department of Physics and Astronomy, University of New Mexico; \\ Albuquerque, New Mexico 87131, USA. \\ ${ }^{3}$ Sandia National Laboratories, Albuquerque, New Mexico 87185, USA.
}

Simulations of granular packings in 2-D by throwing disks in a rectangular die are performed. Different size distributions as bimodal, uniform and gaussian are used. Once the array of particles is done, a relaxation process is carried on using a large-amplitude, low-frequency vertical shaking. This relaxation is performed a number $\mathrm{N}$ of times. Then, we measure the density of the package, contact distribution, coordination number distribution, entropy and also the disks size distribution vs. height. The dependence of all these magnitudes on the number $\mathrm{N}$ of "shakings" used to relax the packing and on the size distribution parameters are explored and discussed.

Keywords: granular packings, simulation.

\section{Introduction}

The characteristics of particle packing are of great interest since the final density obtained upon sintering depends critically on the initial packing. The results obtained from computer simulations of packings of spheres and disks in three and two dimensions, respectively, are important in many areas of science, from powder technology in ceramics to pharmacology improvements [1-3].

In the present work we perform simulations of granular packings in 2-D by throwing disks in a die to represent the actual experiment of poured grains into a rectangular container. There have been previous works where this kind of packings have been generated [4-6]. Typically, the calculated geometrical properties were density, average number of contacts, radial distributions and size distribution of interstices. Other works have dealt with the calculation of the contact force distributions [7-9]. But a complete study of the dependence of all these quantities and others on the size distribution parameters of the grains is still lacking in the literature. To know which are these parameters determining the behavior of the packing when it is put under stress or compaction would be an important goal in the characterization of any granular packing to be used in any technological or industrial application. Here, we will present the first results of a series of computer simulations of packings of disks using different size distributions as bimodal, uniform and gaussian.

\section{Simulation algorithm}

The computer algorithm used in the present simulations has been programed in a way that allows to generate packings of disks sampled from any desired size distribution. The time needed to set a packing of 10,000 disks into a rectangular die of any size is just a few seconds on a Pentium PC. Because of round off errors, the optimum rate between the 


\section{DISCLAIMER}

This report was prepared as an account of work sponsored by an agency of the United States Government. Neither the United States Government nor any agency thereof, nor any of their employees, make any warranty, express or implied, or assumes any legal liability or responsibility for the accuracy, completeness, or usefulness of any information, apparatus, product, or process disclosed, or represents that its use would not infringe privately owned rights. Reference herein to any specific commercial product, process, or service by trade name, trademark, manufacturer, or otherwise does not necessarily constitute or imply its endorsement, recommendation, or favoring by the United States Government or any agency thereof. The views and opinions of authors expressed herein do not necessarily state or reflect those of the United States Government or any agency thereof. 


\section{DISCLAIMER}

Portions of this document may be illegible in electronic image products. Images are produced from the best available original document. 
maximum and minimum disk radii ( $a=R_{\max } / R_{\min }$ ) is up to 20 , i.e., one can generate packings with particles twenty times greater than the smaller ones in it.

A radii distribution for the disks is selected and a number of 10,000 disks are randomly sampled from it. Basically, disks are thrown one at a time from the top, with the horizontal position selected at random but keeping them from overlapping the walls of the container. Each grain falls down following a steepest descent algorithm. Once it touches another disks already deposited, it rolls over them until a stable position is found. By stable we mean the first time the center of the falling particle is in between the center of the two first particles it touches. In the case the stable position is attained where one of the contacting disks has a vertical coordinate for its center that is greater than the corresponding one for the new disk, a bridge position is defined and registred.

If the falling grain touches particles on the bottom of the die, these bottom particles roll on the bottom without friction until the new particle reaches equilibrium on the bottom itself or on other two particles if there is no enough place for it. In this way an ordered bottom is obtained.

Once the packing of particles is ready, a relaxation process is carried out using a largeamplitude, low-frequency vertical shaking [10]. The particles are allowed to fall down again, one at a time. The first to fall are the ones who have lowest vertical positions in the packing. The rules to attain equilibrium are the same as before. This relaxation can be performed a number $\mathrm{N}$ of times. Before relaxing and after each relaxation we measure all the quantities of interest.

In what follows, results for several measured quantities as a function of the radii distribution of the disks and the number of relaxations of the packing are presented.

\section{Results and Discussion}

Three different size distributions are used in the simulations: bimodal, uniform and gaussian. All of them have a mean radius of 10 units. For the first one, the separation between the two peaks is symetrically increased respect to the value of ten and all the data registred for $a$ ranging from 1.1 to 19. The dispersion of the other two types of distributions is increased in such a way that $a$ ranged from 1.2 to 19 . The total number $N$ of shakings studied is $\mathbf{8}$ for each distribution and each $a$ value. The disks fall down into a die of 2000 units width without any restriction on the package height, which is determined once the all of disks are inside the container.

Below is the description of the behavior of the packings for each type of distribution.

Uniform: the top height of the packings does not depend on the number of shakings but does depend on the dispersion of the distribution, as $a$ increases, the top height increases. The fluctuations of the height are higher for small values of $a$. Consequently, the density of the package is slightly smaller for increasing $a$. The number of particles on the bottom was independent of the shaking number for $a \leq 3$ increasing slowly with $N$ for greater values of the dispersion. The mean number of bottom particles is greater for greater values of $a$.

The quantity that is really affected by the relaxation process is the number of bridges, $N_{B}$, in the packing. Figure 1 (a) shows the decay of $N_{B}$ with $N$ for different values of $a$ and different distributions (see below). For high $a, N_{B}$ only decreases for the first shakings, then fluctuating its value.

The mean coordination number is 4 for all the packings independiently of $\mathrm{N}$ and $a$. Typical coordination number distributions, $F$, are shown in Figure 2 (a). 
The orientation of the random packing can be measured through the expected absolute values of the projected length of contacts on a given axis. We measure the projections on the vertical and horizontal axis of the die. Projections do not change with the dispersion of the radii distribution and they are always of the same magnitude, meaning random orientation. There is no dependence on the shaking relaxation. Concerning the angle distribution of normal contacts, no changes with $\mathrm{N}$ are observed, but it changes with $a$. The maximum is more pronounced for greater dispersions and moves from $45^{\circ}-53^{\circ}$ for $a=1$ to $0^{\circ}$ $25^{\circ}$ for $a=19$. The distribution is symetric in the four quadrants.

An interesting parameter to describe the degree of randomness in the distribution of a random variable is the entropy $S$. We define and measure two entropic quantities associated with the coordinate and angle probability distributions, $S_{c}=-p_{i} \ln p_{c i}$ and $S_{a}=-$ $p_{a i} \ln p_{a i}$, respectively, where $p_{c i}$ and $p_{a i}$ are the corresponding probability functions. $i$

Both quantities decrease slightly for the first relaxation shaking but are insensible to the rest of the relaxation process. $S_{c}$ grows continuously with increasing $a$ while $S_{a}$ only increases for $a$ changing from 1 to 1,6 , becoming stable for greater $a$. Only the packings with $a=19$ present a smooth segregation effect due to the shakings performed.

Gaussian: the top height of the packings increases as $a$ increases, being $15 \%$ grater for $a=19$ respect to $a=1.5$. This changes are more pronounced than the corresponding ones for the uniform case. No observable dependence on $\mathrm{N}$ was observed. The behavior of the number of particles on the bottom is the same as that for the uniform case. Figure 1 (b) shows again the decay of the number of bridges, $N_{B}$, with $\mathrm{N}$ for different values of $a$. The number of bridges for the present distribution is of the order of $40 \%$ greater than that for the uniform case for the same $a$. As above, the mean coordination number is 4 for all the packings. Coordination number distributions, $F$; keep the same qualitatively shape as for the uniform case, but here the distributions flatten for all $a$. See Figure $2(b)$.

The projections, angle distributions and entropy quantities behave similarly as for the uniform case. No segregation is present even for high $a$.

Bimodal: there is a slight trend for the density to grow with $a$. This is due to the fact that the smaller disks lie between the bigger ones, improving the packing efficiency. Because the mean radius of the packings increases as $a$ increases for this distribution, one expects that the number of particles on the bottom will decrease with the separation between peaks. This effect is just observed when $a \geq 7$ and the same occurs with the relaxation process which affects the number of bottom particles as we will see below.

As for the previous distributions, the mean coordination number is 4 for all the packings with $a<4$ and decreases for greater values of $a$ as we will see below.

Here, the relaxation process also helps to highly decrease the number of bridges. For $a<8$, the initial number of bridges is comparable to the corresponding ones for previous distributions, but for greater values of $a$ it is considerably higher (almost three times respect to the uniform case) as can be seen in Figure 3.

The distribution of coordination numbers is close to its counterparts for $a<4$. For $a=4,7$ and 19 there is a peak in the distribution for $n=3,2$ and 2 respectivelly. This may be explained if we think that the small particles percolate through the insterticies formed by the bigger ones. Segregation is observed for $a>7$ in coincidence with the increment in the number of bottom particles cited above. 


\section{Conclusions}

In this paper we presented a simulation algorithm to generate random packings of disks in 2-D where the radii size distribution can in principle be any one desired. We got results for uniform, gaussian and bimodal. After the packing is ready, a low-frequency high-amplitude relaxation process is performed. The algorithm is very fast, consuming a few seconds of CPU time.

In general, except for high $a$ in the bimodal case, the mean coordination number is four, independiently of dispersion and relaxation.

The relaxation process is usefull for the rearrangement of the disks in the packing, lowering considerably the number of bridges. It does not practically affect the density of the system and does not cause considerable segregation, except for the bimodal distribution at high $a$. The initial number of bridges is higher for the bimodal distribution compared with the other distributions.

Distributions with greater $a$ values improve the packing efficiency for the first two distributions and make it worse in the bimodal case.

Given the advantages of the present algorithm respect to CPU time and size distributions possibilities, a wide variety of experimental set ups can be simulated in order to predict the force contact distributions before a compressing process is carried on. Present efforts are driven in that direction.

\section{References}

Sandia is a multiprogram laberatory operated by Sandia Corporation, a Lockheed Martin Company, for the United States Department of Energy under contract DE-AC04-94AL85000.

1. H. M. Jaeger, S. R. Nagel and R.P. Behringer, Rev. Mod. Phys. 68 (1996) 1259.

2. A. Mehta, Granular Matter: An Interdisciplinary Approach, Springer-Verlag, New York (1994).

3. V.M. Kenkre, M.R. Endicott, S.J. Glass and A.J. Hurd, J. Am. Ceram. Soc. 79 (1996) 3045.

4. G.T. Nolan and P.E. Kavanagh, Powder Technology 72 (1992) 149; Powder Technology 76 (1993) 301; Powder Technology 78 (1994) 231.

5. J. Grindlay and A.H. Opie, Phys. Rev. E 51 (1995) 718.

6. S. Luding, Phys. Rev. E 55 (1997) 4720.

7. D.C. Hong, Phys. Rev. E 47 (1993) 760.

8. F. Radjai, D.E. Wolf, M. Jean, J.J. Moreau, Phys. Rev. Lett. 80 (1998) 61.

9. S.F. Edwards and D.V. Grinev, Phys. Rev. Lett. 82 (1999) 5397.

10. R. Jullien, P. Meakin, A. Pavlovitch, Phys. Rev. Lett. 69, (1992) 640. 


\section{Figure Captions}

FIGURE 1: Number of bridges $N_{B}$ as a function of the number of "shakings" $N$ and different $a$, (a) for uniform distributions, (b) for gaussian distributions.

FIGURE 2: Typical coordination number distributions, $F$, for (a) uniform and (b) gaussian distributions. $N$ does not affect their shapes.

FIGURE 3: Number of bridges $N_{B}$ as a function of the number of "shakings" $N$ for the bimodal distributions and different $a$. 

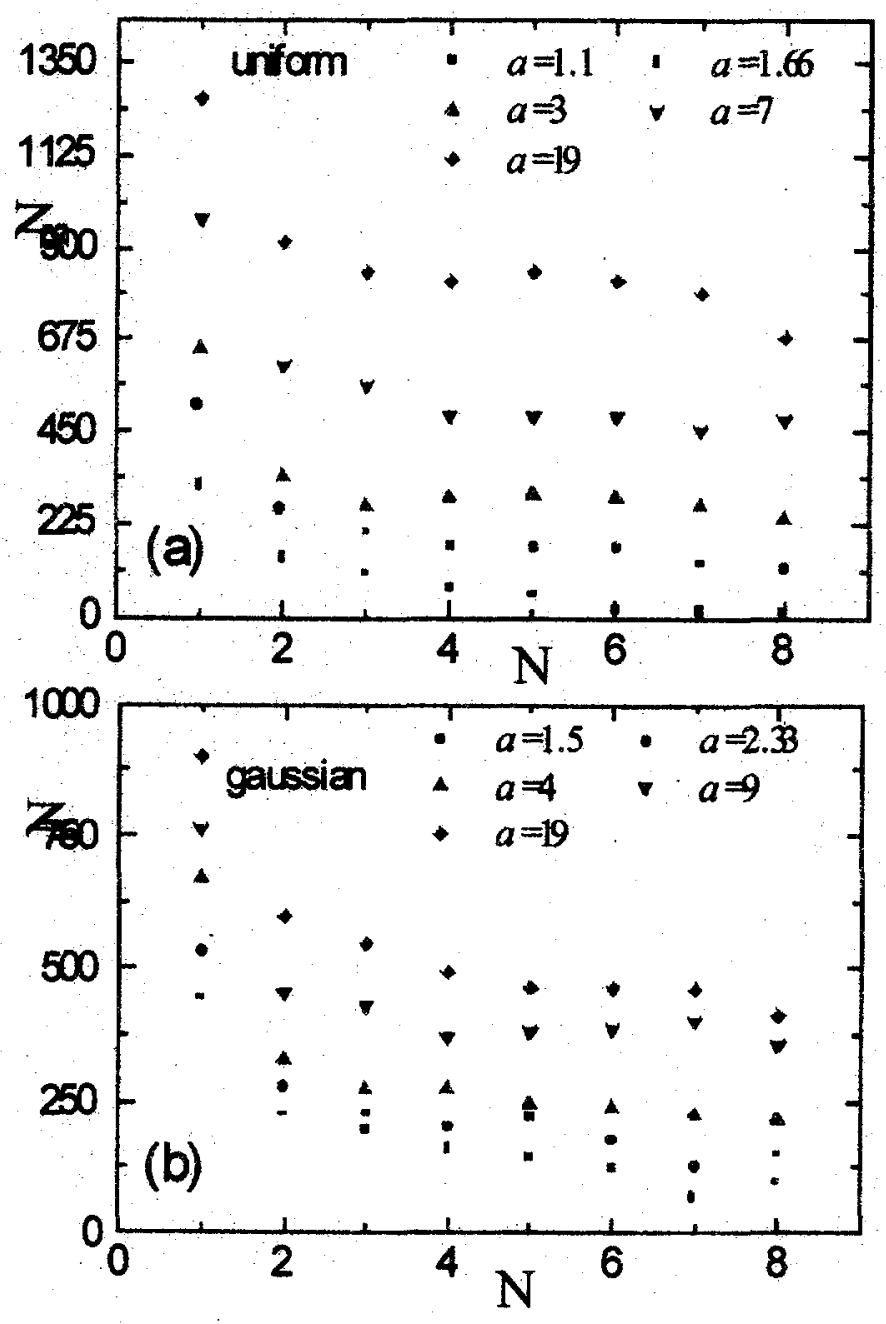

FIGURE 1 

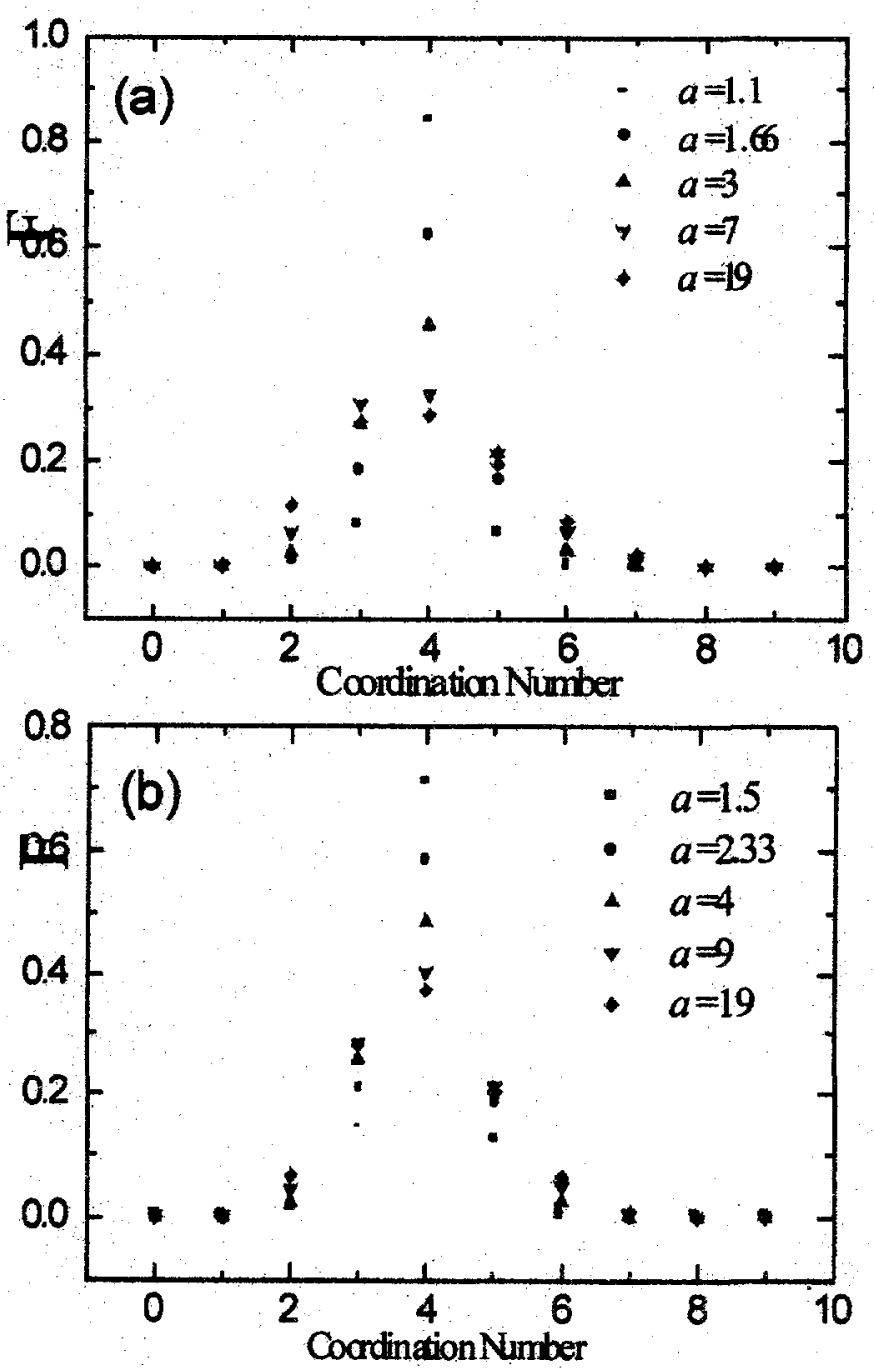

FIGURE 2 


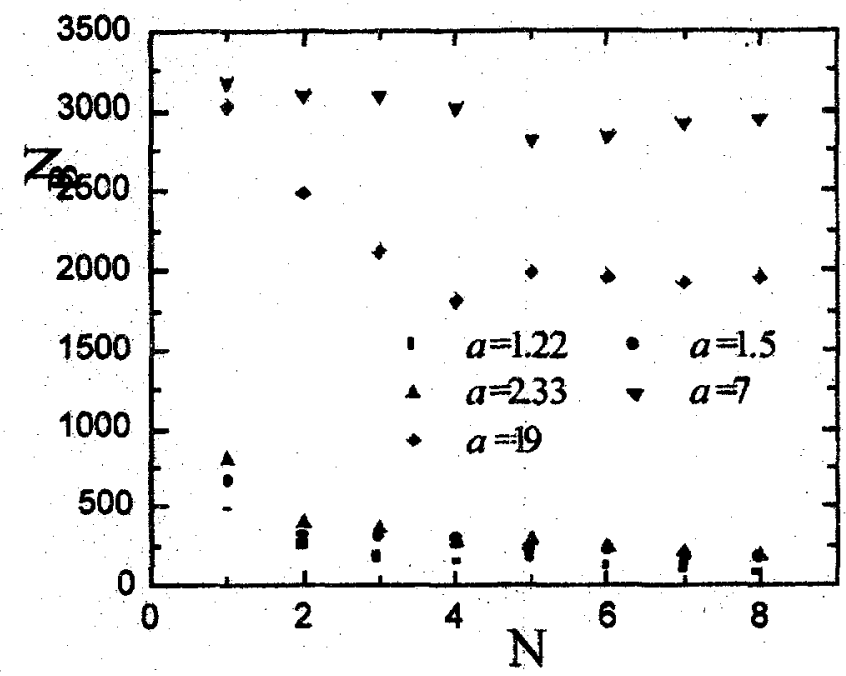

FIGURE 3 